\title{
Transport current loss of BSCCO/Ag tape in different orientations of the external alternating magnetic field
}

\author{
J. J. Rabbers, B. ten Haken and H. H. J. ten Kate \\ University of Twente, Faculty of Applied Physics, Low Temperature Division, P.O. Box 217, 7500 AE Enschede, The Netherlands
}

\begin{abstract}
BSCCO/Ag tapes are being developed for electrical power applications at liquid nitrogen temperatures. In these applications, conductors are exposed to an alternating magnetic field and fed simultaneously with an alternating transport current. In this contribution the influence of an external alternating magnetic field on the transport current loss of a single tape is studied experimentally. Special attention is paid to the loss as a function of the orientation of the external magnetic field. The transport current loss in a DC external magnetic field can be described well by the decrease of the critical current due to the magnetic field. In the case of an $\mathrm{AC}$ external magnetic field this is only a minor effect. Effects responsible for the increase of the transport current loss in external AC magnetic field are investigated with respect to their angle dependency. A model based on a 1 dimensional modelling of the conductor and an average critical current density during the magnetic field cycle is presented. This model describes the measured results reasonably well below the critical current.
\end{abstract}

\section{INTRODUCTION}

When a BSCCO/Ag tape is used in an application such as an electrical power cable or a (transformer) coil, the conductor is exposed to an external AC magnetic field and fed simultaneously with an AC transport current. In general the external field will be much higher than the self-field. On conductor level the total loss can be distinguished in a transport current loss part, energy delivered by the transport current power supply, and a magnetisation loss part, energy delivered by the changing external magnetic field. Depending on the amplitude of the alternating transport current and the external magnetic field, the magnetisation loss or the transport current loss is the dominating loss [1]. In general the magnetic field can have different angles with respect to the tape surface. In motors, generators or in a three-phase power cable a conductor is exposed to a rotating magnetic field.

The increase of the transport current loss caused by an external DC magnetic field is described well by the decrease of the critical current due to the magnetic field. This result holds for all angles of the magnetic field perpendicular to the current [2]. This paper focuses on the transport current loss measurements in external applied alternating magnetic field. Especially the loss as a function of the angle of the magnetic field with respect to the tape is investigated.

For the two limiting cases of alternating magnetic field perpendicular and parallel to the wide side of the tape the results of a transport current experiment are already published [3], [4]. A typical result for magnetic field parallel to the tape is shown in Fig. 1.

For $B_{a}=0 \mathrm{mT}$ the standard transport current loss relation with a slope between +3 and +4 in the double logarithmic plot is observed. When the transport current exceeds the critical current the slope of the loss relation increases due to flux flow. However, when an external alternating magnetic field is applied, loss curves with a slope of +2 are measured below the (field dependent) critical current. The extra loss is caused by an effect that is referred to in literature as the dynamic resistance [5]. Above the critical current the slope of the loss relation again increases due to flux flow. Earlier it is shown the for $B_{a}>\approx 35 \mathrm{mT}$ the loss can be described with a dynamic resistance obtained from the DC voltage current relation in external alternating magnetic field [3].

In case of combined alternating magnetic field and transport current, the influence of a decreased critical current, due to the magnetic field, on the hysteresis loss is only a minor effect [3]. The mechanisms that cause the increase of the transport current loss are investigated with respect to their angle dependency. An angle dependent loss relation including these mechanisms is compared with the measured transport current loss as a function of field angle.

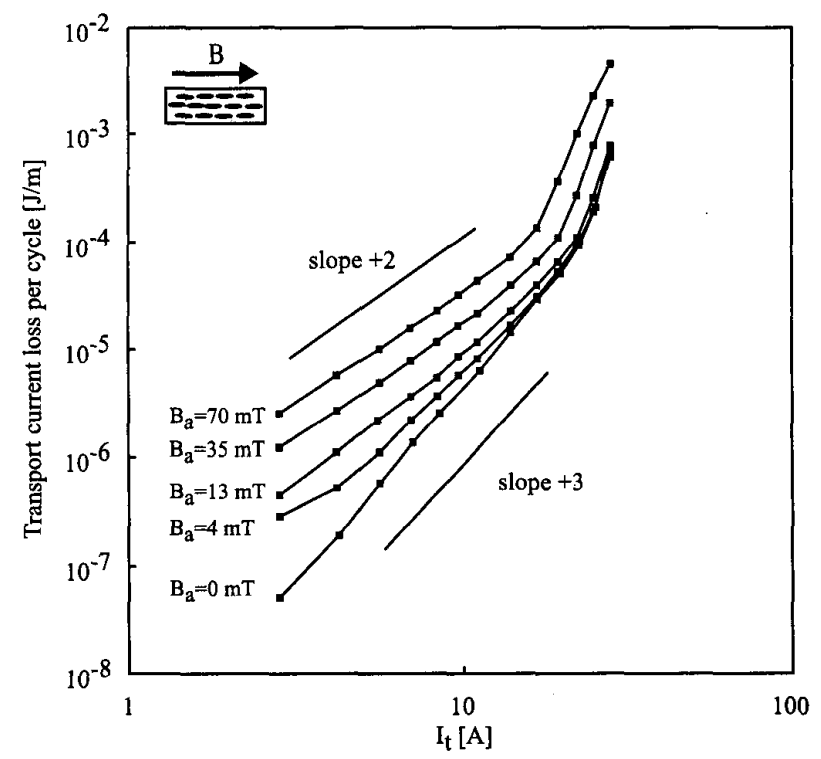

Fig. 1. Transport current loss in external alternating magnetic field parallel to the tape conductor $(\mathrm{T}=77 \mathrm{~K}, \mathrm{f}=35 \mathrm{~Hz})$. 


\section{MODEL}

When a BSCCO/Ag superconducting tape is exposed to an alternating magnetic field and simultaneously fed with an alternating transport current, two sources try to occupy the available (current) space in the conductor. The magnetic field induces shielding currents and the transport current power supply has to change the transport current. The energy that is delivered by the transport current power supply, i.e. the transport current loss, increases when the transport currents interact with the external alternating magnetic field. This increase is presented in Fig. 1. Already for small external magnetic field amplitudes of a few mT's, an increase of the transport current loss is observed.

When the orientation of the magnetic field is changed, two quantities are changed. First the 'dimension' of the conductor perpendicular to the field $d(\alpha)$, see Fig. 2, changes. This effect is modelled with:

$$
d(\alpha) \propto|\cos \alpha|+\frac{b}{a}|\sin \alpha|,
$$

where $\alpha$ is the angle as defined in Fig. 2. The quotient $b / a$ is the inverse of the aspect ratio of the conductor. Equation (1) is a 1-dimensional description of the bar geometry of a $\mathrm{BSCCO} / \mathrm{Ag}$ tape conductor. The two limiting cases of the magnetic field, $\alpha=90^{\circ}$ and $\alpha=0^{\circ}$ are described as an infinite slab in parallel field and an infinitely thin strip in perpendicular field respectively. In this description the multifilament conductor is considered to behave as one homogeneous conductor with completely coupled filaments. For the frequency and field range used in the experiments this description is certainly valid. Also for transport current the multifilament conductor behaves as a fully coupled conductor.

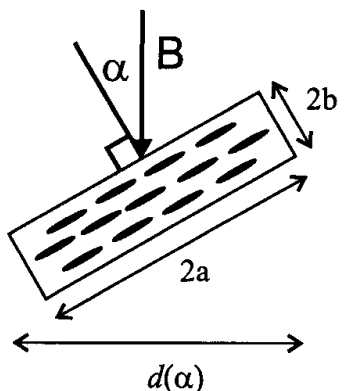

Fig. 2. Cross section of the conductor, definition of the angle $\alpha$.

The critical current density changes also when the orientation of the magnetic field changes. The average critical current density $\bar{J}_{c}\left(B_{a}, \alpha\right)$ over a magnetic field cycle is calculated from DC voltage current measurements [6] with:

$\bar{J}_{c}\left(B_{a}, \alpha\right)=\frac{1}{T} \int_{0}^{T} J_{c}\left(B_{a} \sin (2 \pi f t), \alpha\right) \cdot d t$ where $T$ is the period time of the magnetic field cycle, $B_{a}$ is the amplitude of the magnetic field, $J_{c}$ is the critical current density and $\alpha$ is the angle between magnetic field and the tape.

The total AC loss in case of combined alternating magnetic field and transport current for a slab in parallel field is described in [7]. This analysis is based on the critical state model for an 'infinite' slab. Assuming that the magnetisation loss is not influenced by the alternating transport current [1], a set of angle dependent transport current loss relations containing the angle dependency modelled in (1) and (2) are derived:

$$
\begin{array}{ll}
Q(\alpha) \propto \frac{1}{\bar{J}_{c}\left(B_{a}, \alpha\right)} & \text { for } \beta<i, \\
Q(\alpha) \propto \frac{d(\alpha)}{\bar{J}_{c}\left(B_{a}, \alpha\right)} & \text { for } i<\beta<1 \text { and } \beta>>1,
\end{array}
$$

where $\beta=B_{a} / B_{p}(i=0)$ and $i=I_{t} / I_{c}$ are the normalised magnetic field and transport current amplitude respectively. Fig. 3 shows an overview of the different angle dependent quantities in (1) and (2).

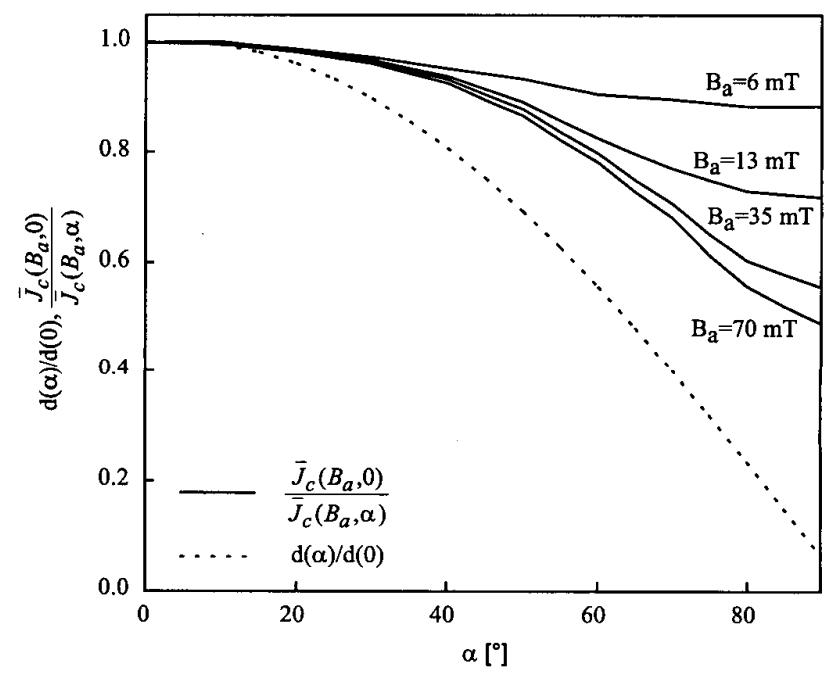

Fig. 3. Overview of the angle dependent quantities.

When the transport current increases to values above the critical current, an additional loss contribution originating from flux flow can be expected. This contribution will also depend on the angle of the magnetic field with respect to the tape because it depends on the, angle dependent, critical current. If the transport current amplitude increases even more, also part of the current flows trough the silver. This gives rise to a resistive loss contribution. Flux flow and resistive losses are not included in the model used in this paper. 


\section{EXPERIMENTAL DETAILS}

The measurements are performed at $77 \mathrm{~K}$ on a 55-filament non-twisted BSCCO-2223 tape superconductor with a pure silver matrix and a critical current density of about $150 \mathrm{~A} / \mathrm{mm}^{2}$. The cross sectional area is $2.9 \mathrm{~mm} \times 0.21 \mathrm{~mm}$ and the superconductor-filling factor is about $25 \%$.

A straight piece of sample is measured in a dipole magnet. The sample can be rotated in the magnetic field. The angle $\alpha$, see Fig. 2, is defined as the angle between the magnetic field and the normal on the wide side of the tape. The sample voltage is measured with a lock-in amplifier. Inductive compensation circuits are used to minimise the voltage induced in the pick-up loop in the voltage taps. Both the external alternating magnetic field and the self-field induce voltages in this pick-up loop. The voltage induced by the external alternating magnetic field is minimised with the ' 8 ' shaped pick-up loop arrangement shown in Fig. 4. The signals induced by the external magnetic field in the two half loops cancel because they have opposite sign. This holds for every angle $\alpha$ between tape and magnetic field. The transport current power supply and the magnet current power supply are decoupled by using a stainless steel tube around the magnet as current return wire. Because the mutual inductance between the two circuits is minimised the two power supplies work almost independently without influencing each other. The magnetic field and the transport current are in-phase. Details of the measurement set-up are described elsewhere [3], [4].

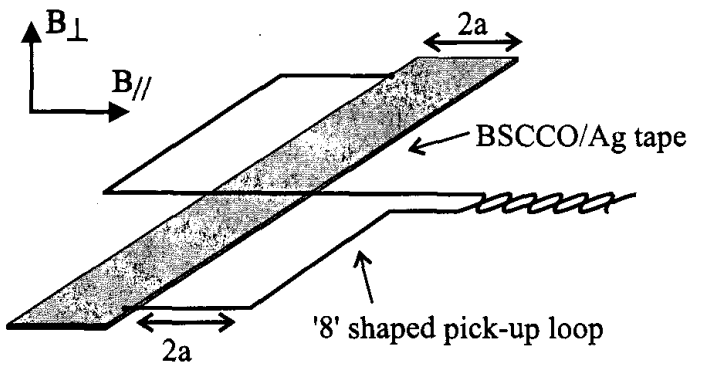

Fig. 4. The ' 8 ' shaped pick-up loop arrangement.

\section{RESULTS}

The normalised transport current loss as a function of the field angle $\alpha$ for constant magnetic field amplitude $\left(B_{a}=70 \mathrm{mT}\right)$ and different transport current amplitudes is shown in Fig. 5. The solid line represents a scaling of the loss as a function of the angle based on (4). In general the model follows the cosine like shape of the measured points. For the largest transport current amplitude $\left(I_{1}=22\right.$ A) a larger deviation between the model and the measurements is observed than for the lower three current amplitudes. The transport current amplitude in this case is remarkably higher than the critical current, so loss contributions from flux flow and/or silver resistance are also present. This situation is not accounted for in the model.

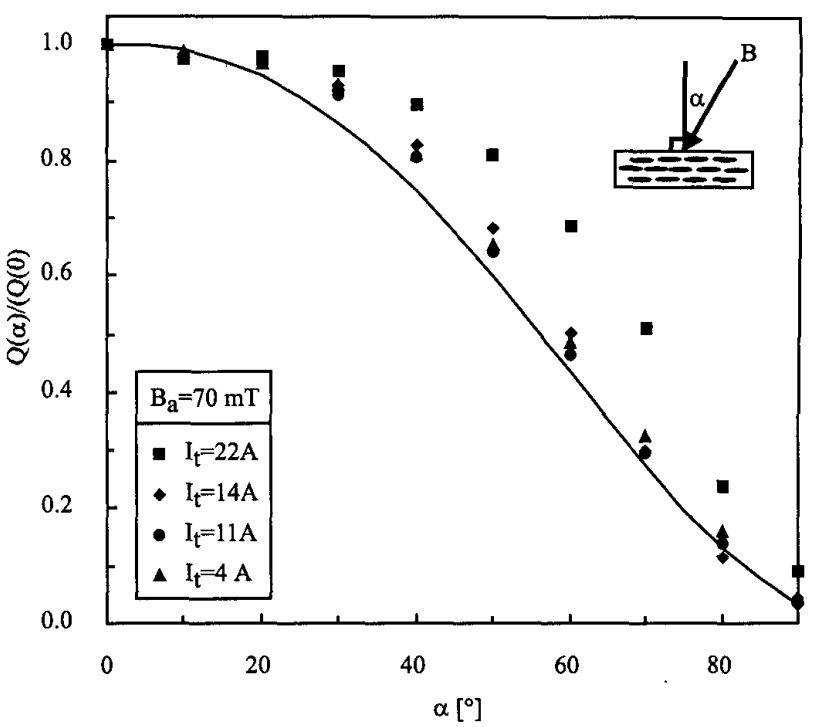

Fig. 5. Normalised transport current loss as a function of field angle $\alpha$ for fixed magnetic field amplitude. The line is based in (4), the symbols are measured points. ( $\mathrm{f}=35 \mathrm{~Hz}, \mathrm{~T}=77 \mathrm{~K}$ )

The lowest transport current amplitude displayed in Fig. $4, I_{1}=4 \mathrm{~A}$, is well below the critical current for the complete range of angles between $0^{\circ}$ and $90^{\circ}$. In this regime no loss contributions from flux flow or silver resistance are expected, the model (4) describes the measured points well. The two series in Fig. 5 for $I=11$ and $I=14$ A also show a good agreement with the model although the critical current is already exceeded slightly during part of the transport current cycle (except for angles $>80^{\circ}$ ). Note that the ratio between the loss for $\alpha=0^{\circ}$ and $\alpha=90^{\circ}$ is about the aspect ratio of the conductor (15).

The normalised transport current as a function of field angle $\alpha$ for constant transport current amplitude and different magnetic field amplitudes is shown in Fig. 6.

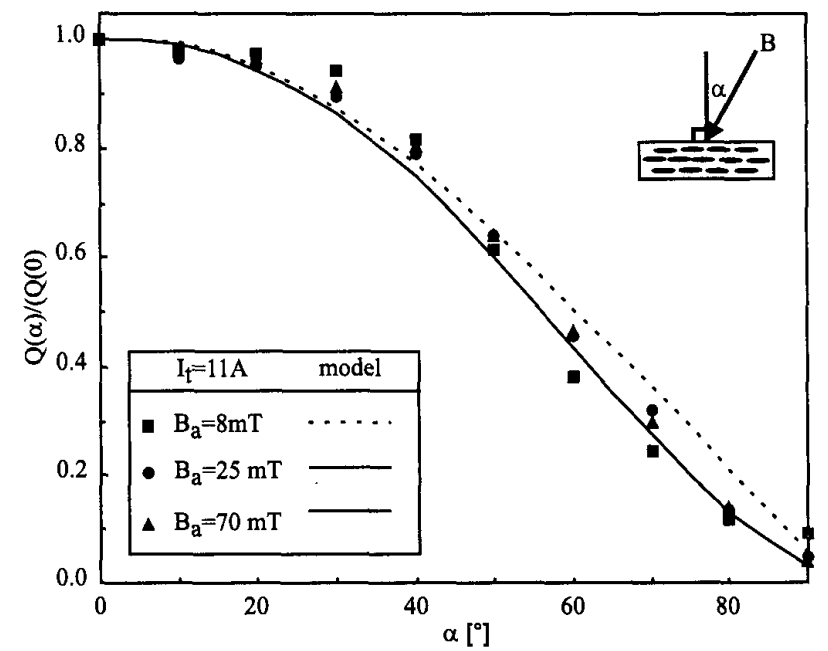

Fig. 6. Normalised transport current loss as a function of field angle for different magnetic field amplitudes and fixed transport current amplitude $(\mathrm{f}=35 \mathrm{~Hz}, \mathrm{~T}=77 \mathrm{~K}$ ). The lines are based on (4), the symbols are measured points. 
Two lines, solid and dashed, represent the model in the picture. The difference between the lines originates from a different $\bar{J}_{c}\left(B_{a}, \alpha\right)$ calculated with (2).

The difference between model lines calculated for $B_{a}=70$ $\mathrm{mT}$ and $B_{a}=25 \mathrm{mT}$ is negligible. In general the measured data follows the cosine like behaviour as the model in (4). In the case of $B_{a}=8 \mathrm{mT}$, a more pronounced reduction of the loss between $\alpha=30^{\circ}$ and $\alpha=70^{\circ}$ is not described by the model. The most probable explanation for this behaviour in this specific field and current amplitude regime $\left(B_{a}=8 \mathrm{mT}\right.$ and $\left.I_{\mathrm{t}}=11 \mathrm{~A}\right)$ is a gradual change to a hysteretic loss behaviour when the angle increases. This situation, $\beta<i$, is described in (3). But, because the ratio between $\beta$ and $i$ changes when the angle increases and only close to $\alpha=90^{\circ}$ the condition of (3) is valid, this situation cannot be modelled with the model presented here.

Summarising, the loss changes due to several angle dependent mechanisms. A distinction is made in regimes, see Fig. 7. In regime I, an extra movement of magnetic flux in the conductor, is responsible for an increase of the transport current loss. This loss is referred to as the 'dynamic resistance loss'. The loss contribution is proportional to the dimension of the conductor perpendicular to the magnetic field, see Fig. 2 , and the critical current. This effect is modelled with (1) and (2).

In regime II a transition to the normal state takes place. A flux flow loss and a (silver) resistance loss for even higher currents dominate the loss in this case. The boundary between the two regimes depends on $B_{a}, I$ and $\bar{J}_{c}\left(B_{a}, \alpha\right)$. The circled area in Fig. 7 shows a third regime. The transport current loss caused by the external alternating magnetic field is proportional to the second power of the transport current amplitude. The hysteresis loss is proportional to the third power of the transport current. In regime III the two mechanisms become of comparable size.

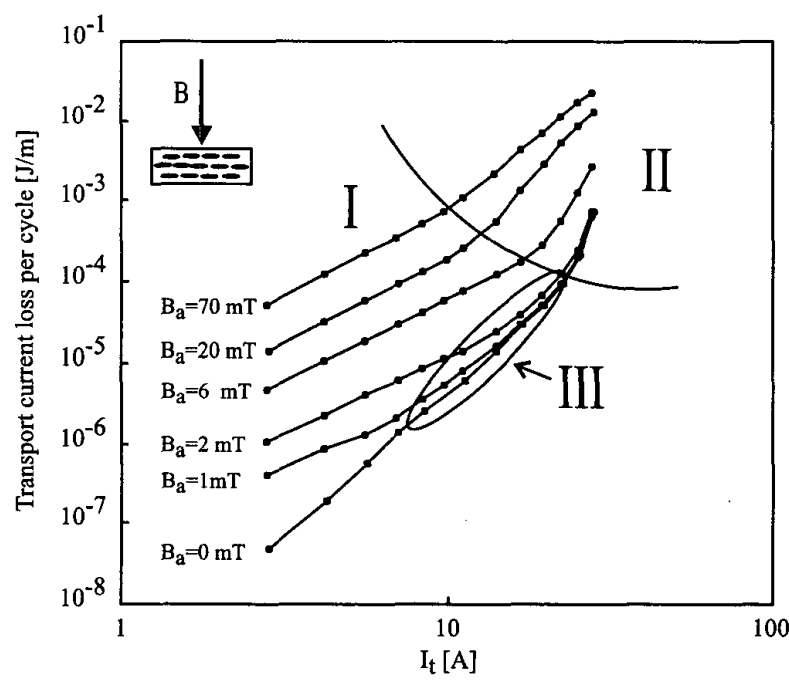

Fig. 7. Transport current loss in external alternating magnetic field, division on different areas $(f=35 \mathrm{~Hz}, T=77 \mathrm{~K})$.
Again this effect is angle dependent. A numerical analysis of the $\mathrm{AC}$ loss for BSCCO/Ag tape in external field with arbitrary angle is presented in [8].

\section{CONCLUSIONS}

The influence of an external applied alternating magnetic field on the transport current loss of a $\mathrm{BSCCO} / \mathrm{Ag}$ tape superconductor is measured. Especially the influence of the angle between the magnetic field and the wide side of the tape is investigated. The lowest loss is obtained for a magnetic field parallel to the wide side of the conductor. For magnetic field perpendicular to the wide side of the conductor the loss is larger. The ratio between the loss due to parallel and perpendicular field is about the aspect ratio of the strip conductor. This approximation holds only when the transport current amplitude is below the, field and angle dependent, critical current. When the transport current amplitude is below the critical current the normalised loss as a function of the angle between conductor and field can be described reasonably well with a scaling relation for the dynamic resistance loss. The effects taken into account are the conductor dimensions perpendicular to the field and the magnetic field dependency of the critical current. When the transport current amplitude exceeds the critical current, deviations between the model and the measurements occur. These deviations are caused by flux flow or resistance loss. For relatively small magnetic field amplitudes and transport current amplitudes close to $I_{\mathrm{c}}$ a situation can occur where the hysteresis loss becomes comparable to the dynamic resistance loss. This results in a different angle dependency.

\section{REFERENCES}

[1] T. Yazawa, J. J. Rabbers, B. ten Haken, H. H. J. ten Kate and Y. Yamada, 'Numerical calculation of current density distributions in HTS tapes with finite thickness in self field and external field," Paper presented at topical ICMC "AC loss \& Stability', 10-13 May 1998 Enschede, The Netherlands (To be published in Physica C).

[2] M. Ciszek, B. A. Glowacki, A. M. Campbell, S. P. Ashworth and W Y. Liang, "Influence of external magnetic field and its orientation on the transport AC losses in Bi-2223 and Tl-1223 silver sheathed tapes," IEEE Transactions on Applied Superconductivity, vol. 7(2), pp. 314-317, June 1997.

[3] J. J. Rabbers, B. ten Haken, F. Gömöry, H. H. J. ten Kate, "Self-field loss of BSCCO/Ag tape in external AC magnetic field," Physica C 300 , pp.1-5, 1998

[4] J. J. Rabbers, B. ten Haken, H. H. J. ten Kate, "Measuring transport current loss of BSCCO/Ag tapes exposed to external AC magnetic field," Paper presented at topical ICMC 'AC loss \& Stability', 10-13 May 1998 Enschede, The Netherlands (To be published in Physica C).

[5] . T. Ogasawara, M. Itoh, Y. Kubota, K. Kanbara, Y. Takahashi, K. Yasohama and $K$. Yasukochi, "Transient field losses in multifilamentary composite conductors carrying transport currents," IEEE Transactions on Magnetics, vol. 17(1), pp. 967-970, 1981.

[6] O. A. Shevchenko, J. J. Rabbers, A. Godeke, B. ten Haken, H. H. J. ten Kate, "AC loss in a high temperature superconducting coil," Paper presented at topical ICMC 'AC loss \& Stability', 10-13 May 1998 Enschede, The Netherlands (To be published in Physica C).

[7] W. J. Carr Jr.,"AC loss and macroscopic theory of superconductors," Gordon and Breach, New York, 1983.

[8] T. Yazawa, J. J. Rabbers, O. A. Shevchenko, B. ten Haken, H. H. J. ten Kate, H. Maeda, "Modeling the current distribution in HTS tapes with transport current and applied magnetic field," presented at ASC '98 Palm Desert. 